Marjory Stephenson Prize Lecture 2010

\author{
Correspondence \\ Jan Tommassen \\ j.p.m.tommassen@uu.nl
}

\section{Assembly of outer-membrane proteins in bacteria and mitochondria}

\author{
Jan Tommassen
}

\author{
Department of Molecular Microbiology and Institute of Biomembranes, Utrecht University, \\ Padualaan 8, 3584 CH Utrecht, The Netherlands
}

\begin{abstract}
The cell envelope of Gram-negative bacteria consists of two membranes separated by the periplasm. In contrast with most integral membrane proteins, which span the membrane in the form of hydrophobic $\alpha$-helices, integral outer-membrane proteins (OMPs) form $\beta$-barrels. Similar $\beta$-barrel proteins are found in the outer membranes of mitochondria and chloroplasts, probably reflecting the endosymbiont origin of these eukaryotic cell organelles. How these $\beta$-barrel proteins are assembled into the outer membrane has remained enigmatic for a long time. In recent years, much progress has been reached in this field by the identification of the components of the OMP assembly machinery. The central component of this machinery, called Omp85 or BamA, is an essential and highly conserved bacterial protein that recognizes a signature sequence at the $\mathrm{C}$ terminus of its substrate OMPs. A homologue of this protein is also found in mitochondria, where it is required for the assembly of $\beta$-barrel proteins into the outer membrane as well. Although accessory components of the machineries are different between bacteria and mitochondria, a mitochondrial $\beta$-barrel OMP can be assembled into the bacterial outer membrane and, vice versa, bacterial OMPs expressed in yeast are assembled into the mitochondrial outer membrane. These observations indicate that the basic mechanism of OMP assembly is evolutionarily highly conserved.
\end{abstract}

\section{Introduction}

The cell envelope of Gram-negative bacteria is composed of two membranes, the inner membrane and the outer membrane, which are separated by the periplasm containing the peptidoglycan layer. While the inner membrane is a phospholipid bilayer constituted of glycerophospholipids, the outer membrane is highly asymmetrical, containing glycerophospholipids in the inner leaflet and lipopolysaccharides (LPSs) exposed to the cell surface (Fig. 1). The outer membrane functions as a permeability barrier protecting the bacteria against harmful compounds, such as antibiotics and bile salts, from the environment. Most nutrients pass this barrier via a family of integral outer-membrane proteins (OMPs), collectively called porins (Fig. 1). These trimeric proteins form open, water-filled channels in the outer membrane, which allow for the passage of small hydrophilic solutes, such as amino acids and monosaccharides, via passive diffusion (Nikaido, 2003). Other OMPs have more specialized transport functions, such as the secretion of proteins and the extrusion of drugs, or function as enzymes or structural components of the outer membrane (Koebnik et al., 2000). Besides integral OMPs, the membrane also

Abbreviations: LPS, lipopolysaccharide; OMP, outer-membrane protein; POTRA, polypeptide transport-associated [domain]; PPlase, peptidylprolyl cis/trans isomerase. contains lipoproteins, which are attached to the membrane via an N-terminal lipid moiety.

All constituents of the outer membrane are synthesized in the cytoplasm or at the inner leaflet of the inner membrane. An area of intense research is how these components are transported and assembled into the outer membrane. An obvious model organism to study such fundamental questions is Escherichia coli, but Neisseria meningitidis has also proven to be a very suitable organism to address these questions. N. meningitidis normally resides as a commensal in the nasopharynx but occasionally causes sepsis and meningitis. Besides generally useful features, such as a relatively small genome size ( 2200 genes $)$ and natural competence and recombination proficiency, which facilitate the construction of mutants, the organism has several properties particularly useful for the study of outer membrane biogenesis. Firstly, in contrast with E. coli, N. meningitidis is viable without LPS (Steeghs et al., 1998). Such mutants defective in LPS biosynthesis still produce an outer membrane into which OMPs are assembled (Steeghs et al., 2001). Since N. meningitidis is viable without LPS, the genes encoding the components of the LPS transport route can be knocked out and the properties of such mutants can be studied (Bos et al., 2004; Tefsen et al., 2005). Secondly, studies on OMP assembly in E. coli are thwarted by a stress response that is activated when unfolded OMPs accumulate 
in the periplasm. Activation of this stress response, which is dependent on the alternative $\sigma$ factor $\sigma^{\mathrm{E}}$, results in the increased production of periplasmic chaperones that aid in OMP assembly and of the protease DegP that degrades these unfolded OMPs (Ruiz \& Silhavy, 2005). In addition, small regulatory RNAs are produced that inhibit the translation of the mRNAs for OMPs by stimulating their decay (Johansen et al., 2006; Papenfort et al., 2006). Thus, OMP synthesis is inhibited under these conditions until unfolded OMPs are cleared from the periplasm. Consequently, mutations resulting in OMP assembly defects do not normally result in the extensive accumulation of unfolded OMPs in the periplasm, but in decreased OMP levels (Chen \& Henning, 1996; Sklar et al., 2007b). Since other signals such as altered LPS structure (Tam \& Missiakas, 2005), and even cytoplasmic signals (Costanzo \& Ades, 2006) can also trigger the $\sigma^{\mathrm{E}}$ dependent stress response, decreased OMP levels do not necessarily reflect an OMP assembly defect. Since this $\sigma^{\mathrm{E}}$ dependent stress response is absent in N. meningitidis (Bos et al., 2007a), unfolded OMPs normally accumulate in the periplasm of assembly-defective $N$. meningitidis mutants, which facilitates these studies. This paper focuses on the current knowledge of OMP biogenesis in bacteria and on the evolutionary conservation of the OMP assembly machinery.

\section{Structure of bacterial OMPs}

Whereas most integral membrane proteins, including bacterial inner-membrane proteins, span the membrane in the form of $\alpha$-helices entirely composed of hydrophobic amino acids, bacterial OMPs present an entirely different structure (Fig. 1). These proteins form $\beta$-barrels composed of antiparallel amphipathic $\beta$-strands (Koebnik et al., 2000).
The hydrophobic residues in these $\beta$-strands are exposed to the lipid environment of the membrane, whereas the hydrophilic residues point towards the interior of the protein, which is the aqueous channel in the case of porins. These $\beta$-barrel structures are very stable, usually withstanding incubation in $2 \%$ SDS (i.e. as present in standard sample buffer for SDS-PAGE) at ambient temperature. This property explains the heat-modifiable behaviour of many OMPs in SDS-PAGE analysis: the native form of these proteins migrates differently in the gel compared with the heat-denatured form (Dekker et al., 1995; Nakamura \& Mizushima, 1976). Also, natively folded OMPs are usually highly resistant to proteases. Heat modifiability and protease resistance are facile parameters to probe the folding of OMPs into their native configuration.

\section{Transport of OMPs across the bacterial inner membrane}

The unusual structure of bacterial OMPs is probably imposed by their biogenesis pathway. OMPs are synthesized in the cytoplasm as precursors with an $\mathrm{N}$-terminal signal sequence, which marks them for transport across the inner membrane via the Sec system (Fig. 2). The proteinconducting channel of the Sec system, which is composed of the integral membrane proteins SecY, SecE and SecG (Driessen \& Nouwen, 2008), releases OMPs and periplasmic proteins at the periplasmic side of the membrane. The SecYEG translocon is also implicated in the assembly of integral inner-membrane proteins. When large hydrophobic protein segments are inserted into the translocon, the channel opens laterally to allow for the insertion of these proteins into the inner membrane (Fig. 2; Driessen \&

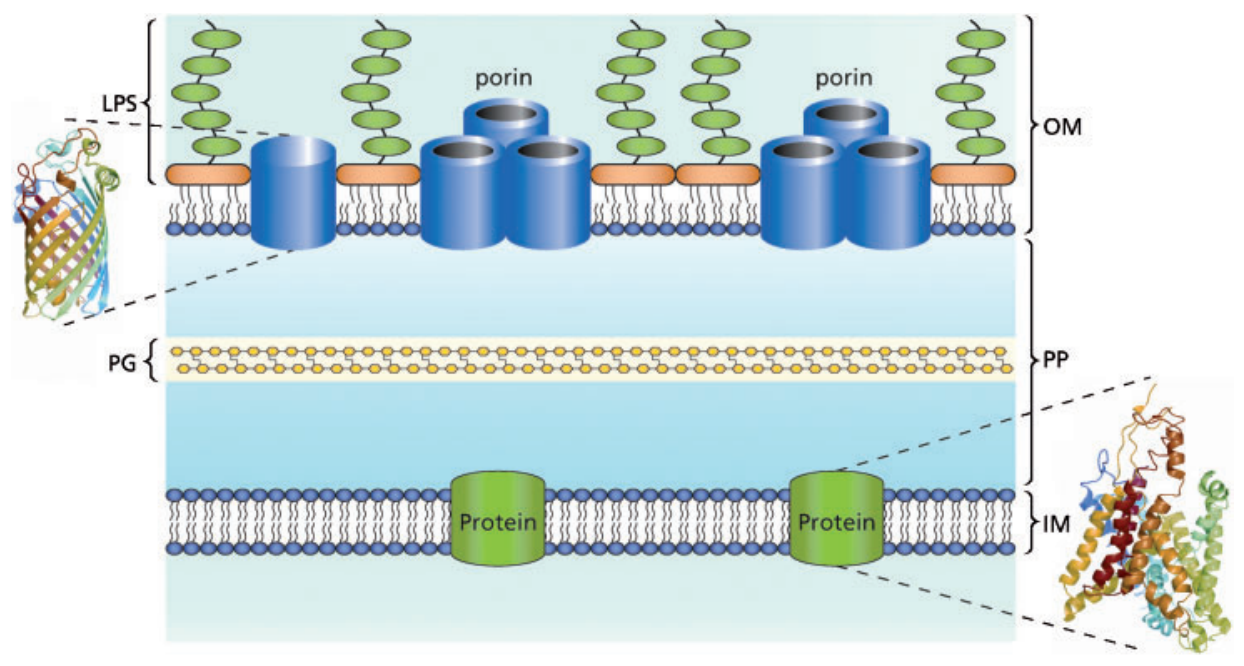

Fig. 1. Structure of the Gram-negative bacterial cell envelope. OM, Outer membrane containing LPS in the outer leaflet of the bilayer and porins as the major protein components; PP, periplasm containing the peptidoglycan layer (PG); IM, inner membrane. Examples of a typical $\beta$-barrel structure of an OMP, i.e. the LPS deacylase LpxR from Salmonella typhimurium (PDB file 3FID) (Rutten et al., 2009), and of a typical $\alpha$-helical inner-membrane protein, i.e. the SecYE translocon of Thermus thermophilus (PDB file 2ZQP) (Tsukazaki et al., 2008), are shown on the left and the right, respectively. 


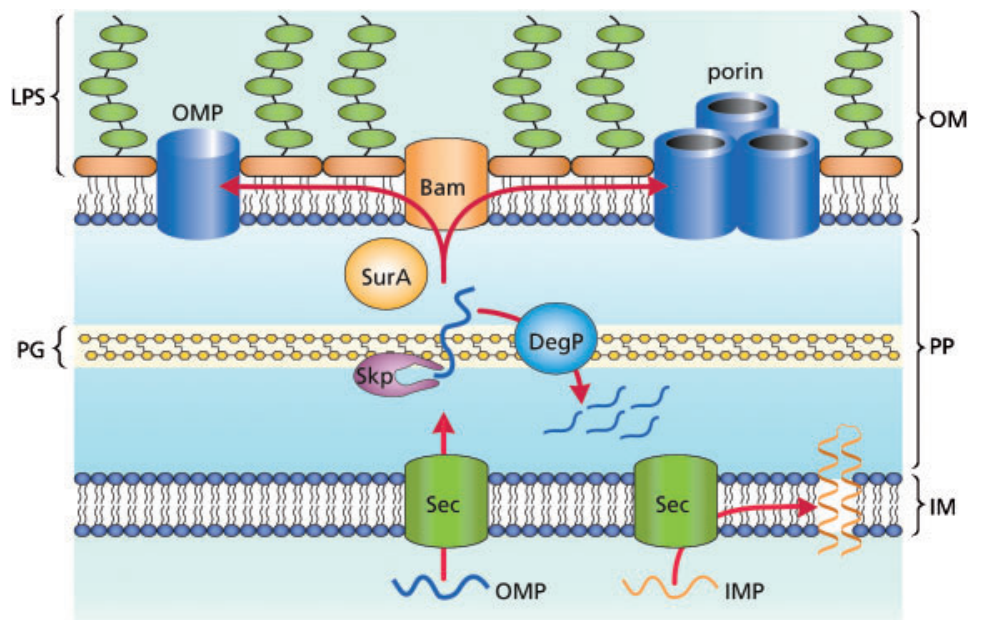

Fig. 2. Model for the biogenesis of bacterial OMPs. Porins and other OMPs are synthesized in the cytoplasm as precursors with a signal sequence, which is cleaved off during or immediately after their transport to the periplasm via the Sec translocon. While still engaged with the Sec translocon, the nascent OMPs are bound by the chaperone Skp, which prevents their aggregation in the periplasm. Folding is initiated when they arrive at the Bam complex in the outer membrane and is, at least for some OMPs, aided by the chaperone SurA. The Bam complex mediates their assembly into the outer membrane. How exactly the nascent OMPs pass the peptidoglycan layer is unknown, but the Bam complex components extend into the periplasm (Fig. 3a, b) and some of them might modulate the peptidoglycan to facilitate the passage of the OMPs. The main function of DegP is probably the degradation of misfolded OMPs. The Sec complex also processes nascent inner-membrane proteins (IMPs) and opens laterally to insert them into the inner membrane. OM, PP, PG and IM are defined in the legend to Fig. 1.

Nouwen, 2008). Thus, the presence of similar hydrophobic segments in OMPs would prevent them from reaching their final destination, while the amphipathic $\beta$-strands that constitute the transmembrane segments of OMPs are compatible with transport via the SecYEG translocon to the periplasm. Indeed, the insertion of hydrophobic segments into the outer membrane porin PhoE of E. coli was shown to affect the biogenesis of the protein (Agterberg et al., 1990).

\section{Transport of OMPs through the periplasm}

In $E$. coli, three chaperones have been reported to guide nascent OMPs during their intermediate periplasmic stage (Fig. 2): Skp, SurA and the protease DegP, which also has chaperone qualities (Spiess et al., 1999). Recent structural analysis showed that DegP in its activated state can form large oligomeric cage-like structures of 12 or 24 subunits that could harbour a folded OMP in its central cavity without degrading it (Krojer et al., 2008). None of these chaperones is essential in E. coli, but double mutants show synthetic, often lethal, phenotypes, suggesting redundancy in chaperone activities. Detailed analyses of single and double mutants suggested the existence of two parallel pathways of chaperone activity in the periplasm, a major SurA-dependent route and an alternative Skp- and DegPdependent route that deals with substrates that fall off the SurA pathway (Rizzitello et al., 2001; Sklar et al., 2007b). However, $s k p$ and $\operatorname{degP}$ mutations have also been reported to show a synthetic phenotype (Schäfer et al., 1999), which is inconsistent with the idea that these chaperones operate within the same pathway. Furthermore, a recent proteomic analysis indicated that SurA has only a few substrates, including the OMP LptD, which is involved in LPS biogenesis, and that the reduced levels of many other OMPs in surA mutants may be solely a consequence of activation of the $\sigma^{\mathrm{E}}$-dependent stress response (Vertommen et al., 2009). The study of Vertommen and colleagues argues against the hypothesis that the SurA pathway is the major periplasmic chaperone pathway for OMPs in the periplasm.

An alternative explanation for the synthetic phenotypes of double chaperone mutants is that these proteins have different, but complementary functions (Bos et al., 2007a; Walther et al., 2009b). Skp selectively binds unfolded OMPs (Chen \& Henning, 1996; de Cock et al., 1999), presumably while they are still engaged with the Sec translocon (Harms et al., 2001). The crystal structure of this trimeric protein has been solved (Korndörfer et al., 2004; Walton \& Sousa, 2004); it resembles a jellyfish that can hold nascent OMPs between its tentacles, thereby preventing their aggregation in the aqueous environment of the periplasm (Walton et al., 2009). SurA appears to play a role in the folding of OMPs into their native configuration (Lazar \& Kolter, 1996; Rouvière \& Gross, 1996). SurA is a peptidyl-prolyl cis/trans isomerase (PPIase) with two PPIase domains, which, however, appear to be dispensable for the chaperone qualities of the protein (Behrens et al., 2001). In this model, Skp is a 'holding chaperone' that prevents folding and aggregation of OMPs in the periplasm, whereas SurA acts as a 'folding chaperone' that assists in the folding of OMPs once they arrive at the assembly machinery in the outer membrane. 
The synthetic lethality of a skp surA double mutant is explained by an increased requirement for a holding chaperone when the folding of the OMPs is compromised by the absence of SurA, and, vice versa, efficient folding is increasingly important when the holding chaperone Skp is absent. The main role of DegP in this model is to prevent toxic accumulation of misfolded OMPs in the periplasm, either by degrading them (Fig. 2) or by sequestering them within the multimeric cage, thereby preventing them from engaging with the assembly machinery in the outer membrane (Bos et al., 2007a; Walther et al., 2009b). Obviously, this role of DegP becomes more important when the activity of Skp or SurA is compromised.

The role of the periplasmic chaperones has also been studied in N. meningitidis, where the $\sigma^{\mathrm{E}}$-dependent stress response is absent (E. Volokhina, M.P. Bos \& J. Tommassen, unpublished results). An important role for Skp in OMP biogenesis in this organism has been confirmed. However, inactivation of the surA gene had no notable effect on OMP assembly; this is consistent with the aforementioned proteomics study in E. coli (Vertommen et al., 2009), which suggested that SurA has only a very restricted number of substrates. Furthermore, inactivation of surA in an $s k p$ mutant of $N$. meningitidis did not aggravate the OMP assembly defect of the skp single mutant. A homologue of DegP is non-existent in N. meningitidis, but there is a homologue of the closely related protease DegQ (Bos et al., 2007a). Inactivation of this $\operatorname{deg} Q$ gene caused no OMP assembly defect and again no synthetic phenotype was observed when the mutation was combined with an $s k p$ or surA mutation (E. Volokhina, M.P. Bos \& J. Tommassen, unpublished results). Thus, at least in $N$. meningitidis, Skp appears to be the major periplasmic chaperone involved in OMP biogenesis.

\section{The bacterial OMP assembly machinery}

After travelling through the periplasm and reaching the outer membrane, OMPs have to fold and insert into this membrane. The first component of the OMP assembly machinery identified was a protein known as Omp85 in $N$. meningitidis. Homologues of Omp85 were identified in all available Gram-negative bacterial genome sequences (Voulhoux et al., 2003; Voulhoux \& Tommassen, 2004), and previous attempts to inactivate the gene in Haemophilus ducreyi and Synechocystis sp. were reported to be unsuccessful (Reumann et al., 1999; Thomas et al., 2001), suggesting an important function for the protein. Furthermore, the omp85 gene was found to be located in many genome sequences immediately upstream of the $s k p$ gene encoding the periplasmic OMP chaperone, suggesting that Omp85 might be involved in OMP biogenesis as well. To assess the function of Omp85, the gene was cloned under an IPTGinducible promoter (Voulhoux et al., 2003). In the absence of IPTG, the resulting mutants stopped growing and all OMPs examined were found to accumulate as unfolded proteins as shown (amongst other characteristics) by their protease sensitivity and their lack of heat modifiability.
These results demonstrated an essential role of Omp85 in OMP assembly.

Non-denaturing SDS-PAGE (Voulhoux et al., 2003) and cross-linking experiments (Manning et al., 1998) indicated that Omp85 is part of a multi-subunit complex in $N$. meningitidis. These results were confirmed in E. coli, where the Omp85 homologue is now called BamA (Bam stands for $\beta$-barrel assembly machinery). BamA forms a complex with four lipoproteins, BamB-E (Fig. 3a) (Wu et al., 2005; Sklar et al., 2007a). Whereas Omp85/BamA homologues are present in all Gram-negative bacteria, the accessory lipoproteins are less well conserved. For example, in the $N$. meningitidis Bam complex, the BamB component is lacking and this complex contains an additional component, RmpM, an OMP with a peptidoglycan-binding motif (Fig. 3b) (Volokhina et al., 2009). In the case of Caulobacter crescentus, the BamC component is absent and a different protein with a peptidoglycan-binding motif, the lipoprotein $\mathrm{Pal}$, is present as an additional component (Anwari et al., 2010). In some alphaproteobacteria, both BamB and BamC appear to be absent (Gatsos et al., 2008). Also, the function of the accessory lipoproteins is less vital. In E. coli, BamD is the only essential lipoprotein component of the complex, whereas mutational loss of the other lipoproteins causes only mild OMP assembly defects (Malinverni et al., 2006; Sklar et al., 2007a). However, even in the closely related bacterium Salmonella enterica, BamD appears to be dispensable (Fardini et al., 2009). Also, in Neisseria gonorrhoeae, a viable knockout mutant in the bamD homologue, designated comL, has been described (Fussenegger et al., 1996) but the gene appears essential for viability and OMP assembly in N. meningitidis (Volokhina et al., 2009). Thus, the Bam complex in bacteria consists of one essential central component, Omp85/BamA, and a variable number of accessory components, the importance of which is variable and depends on the specific component and the bacterium being studied.

\section{Interaction of substrate OMPs with BamA/Omp85}

Electrophysiological experiments demonstrated that purified BamA reconstituted into planar lipid bilayers forms narrow ion-conductive channels (Robert et al., 2006; Stegmeier \& Andersen, 2006). The physiological significance of these channels is still unclear, but this property could be used to study the interaction of the protein with its substrate OMPs. Addition of denatured OMPs to BamA-containing planar lipid bilayers increased the conductivity of the pores, demonstrating a direct interaction between BamA and its substrates (Robert et al., 2006). Since addition of periplasmic proteins to the bilayers had no such effect, this interaction between BamA and its substrates was specific.

The specificity of the interaction between BamA and its substrates indicated the presence of a recognition signal within these substrates. Previously, a signature sequence was recognized at the $\mathrm{C}$ terminus of the vast majority of bacterial OMPs (Struyvé et al., 1991). This signature consists of a phenylalanine (or occasionally tryptophan) at the 
(a)

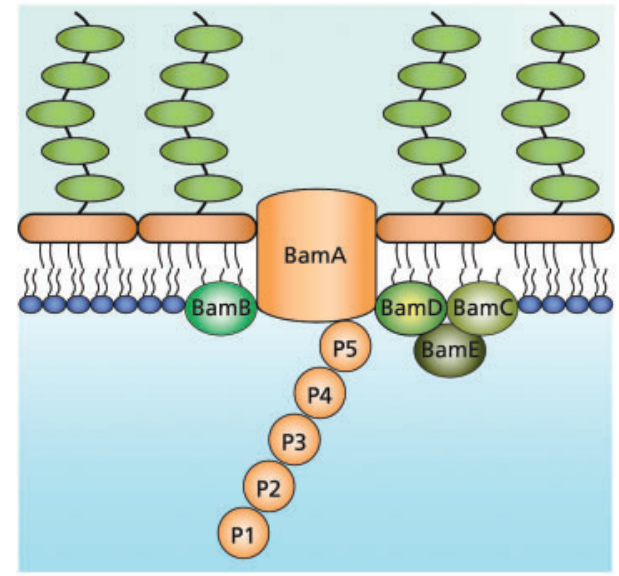

(b)

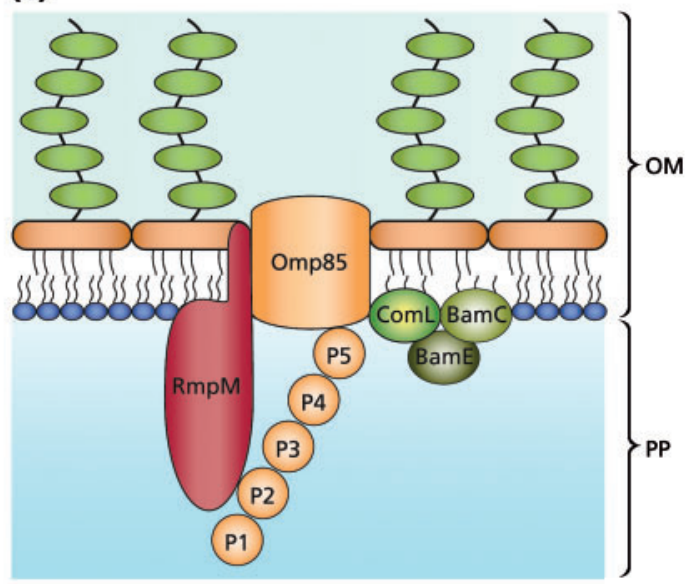

(c)

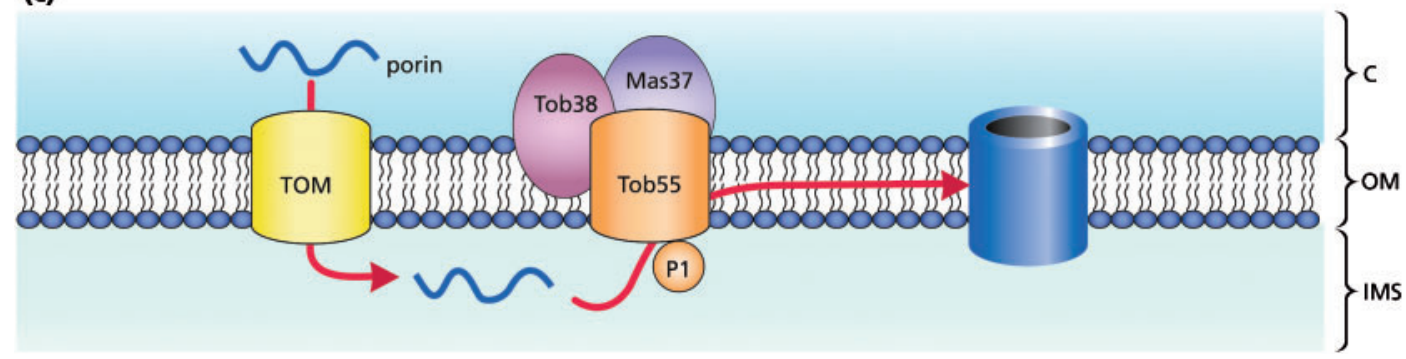

Fig. 3. Comparison of the composition of the $\beta$-barrel OMP assembly complexes in (a) E. coli, (b) N. meningitidis and (c) mitochondria. Homologous components in the various systems are coloured identically. The $\mathrm{N}$-terminal periplasmic part of the bacterial BamA/Omp85 consists of five POTRA domains (P1-P5), whereas the mitochondrial homologue Tob55 contains only one such domain $(\mathrm{P} 1)$. In mitochondria, the precursors of $\beta$-barrel OMPs, such as mitochondrial porin, are first imported from the cytoplasm (C) of the eukaryotic cell via the TOM complex into the intermembrane space (IMS) before they are assembled via the TOB complex into the outer membrane (OM). OM and PP are defined in the legend to Fig. 1.

C-terminal position, a tyrosine or a hydrophobic residue at position -3 relative to the $\mathrm{C}$ terminus, and also hydrophobic residues at positions $-5,-7$ and -9 from the $C$ terminus. Furthermore, the importance of the $\mathrm{C}$-terminal Phe in vivo was demonstrated by its deletion or substitution in porin PhoE (Struyvé et al., 1991). Such mutations severely affected the assembly of the protein into the outer membrane. Of note, however, is that Phe was not absolutely essential: while a mutant protein deleted for the C-terminal Phe accumulated in periplasmic inclusion bodies when it was highly expressed (Struyvé et al., 1991), it was still assembled into the outer membrane when expression levels were reduced (de Cock et al., 1997). This observation could be explained if the mutation decreases but does not abrogate the recognition of the mutant protein by the assembly machinery resulting in its periplasmic aggregation. So, reduced expression will decrease the aggregation kinetics, thereby increasing the time span needed for the assembly machinery to deal with the suboptimal mutant protein.

The hypothesis that the C-terminal Phe is part of the recognition signal for BamA was confirmed in planar lipid bilayer experiments with reconstituted BamA (Robert et al.,
2006). In contrast with wild-type PhoE, the mutant protein lacking the $\mathrm{C}$-terminal Phe did not stimulate the conductivity of the BamA channels. However, at higher concentrations, it blocked the BamA channels, indicating that it can still interact with BamA but differently from the wild-type protein. The latter result indicates that either the recognition signal is not completely disrupted by the deletion or the PhoE protein contains additional signals. This is consistent with the observation that a mutant protein lacking the $\mathrm{C}$ terminal Phe can still be assembled in vivo if the expression level is low (de Cock et al., 1997). The existence of a Cterminal recognition signal in PhoE was further confirmed by using synthetic peptides (Robert et al., 2006). Like the full-length PhoE, a synthetic peptide comprising its last 12 aa stimulated the conductivity of the BamA channels, while control peptides did not.

Omp85/BamA was predicted to consist of two domains, an $\mathrm{N}$-terminal periplasmic domain and a C-terminal domain embedded as a $\beta$-barrel into the outer membrane (Fig. 3a and b) (Voulhoux et al., 2003). The periplasmic part was predicted to consist of five repeated domains, named polypeptide transport-associated (POTRA) domains 
(Sánchez-Pulido et al., 2003). Considering their periplasmic location, it seems likely that these POTRA domains interact with the substrate OMPs. The structures of BamA fragments containing several POTRA domains have been solved by Xray crystallography (Kim et al., 2007; Gatzeva-Topalova et al., 2008) and NMR spectroscopy (Knowles et al., 2008). Although these domains show only very limited sequence identity, they have a common structure consisting of a threestranded $\beta$-sheet overlaid with two $\alpha$-helices. It was suggested that these POTRA domains interact with the substrate OMPs and/or with the accessory lipoproteins of the Bam complex by $\beta$-augmentation (Kim et al., 2007). NMR experiments indeed revealed that several peptides derived from porin PhoE could weakly bind to either side of the $\beta$-sheets in the POTRA domains (Knowles et al., 2008). Unfortunately, a Cterminal fragment of PhoE could not be tested in those experiments because of solubility problems.

\section{OMP biogenesis in mitochondria}

Other than in the outer membranes of Gram-negative bacteria, integral $\beta$-barrel membrane proteins are also found in the outer membranes of mitochondria and chloroplasts, probably reflecting the endosymbiont origin of these eukaryotic cell organelles. It should be noted that these organelles also contain $\alpha$-helical OMPs (Walther \& Rapaport, 2009), which will not be discussed further here. Soon after the discovery that Omp85/BamA is an essential component of the bacterial OMP assembly machinery (Voulhoux et al., 2003), several research groups identified a homologue in mitochondria and showed that it is involved in the assembly of $\beta$-barrel proteins into the mitochondrial outer membrane (Gentle et al., 2004; Kozjak et al., 2003; Paschen et al., 2003). This protein was named either Omp85, Sam50 or Tob55, and will be referred to from here on as Tob55. Tob55 was shown to be part of a complex (called the TOB or SAM complex) with at least two other proteins, which are known under various names, i.e. Tob38/Sam35 and Mas37/Tom37/Sam37 (Fig. 3c) (Wiedemann et al., 2003; Ishikawa et al., 2004; Milenkovic et al., 2004; Waizenegger et al., 2004). These accessory components are exposed to the cytosolic side of the outer membrane and show no homology to the lipoprotein components of the bacterial Bam complex.
Much of the genome of the endosymbiont that evolved into mitochondria has been transferred to the nucleus. Consequently, most mitochondrial proteins are synthesized in the cytoplasm of the eukaryotic cell from where they are transported into the mitochondria via the TOM complex in the outer membrane and the TIM complexes in the inner membrane (Chacinska et al., 2009). Also the precursors of $\beta$ barrel OMPs are synthesized in the cytoplasm from where they have direct access to the mitochondrial outer membrane. Nevertheless, these proteins are first imported via the TOM complex into the intermembrane space of the mitochondria (i.e. the equivalent of the bacterial periplasm) (Rapaport \& Neupert, 1999; Krimmer et al., 2001; Model et al., 2001) to approach the assembly machinery from the same side as occurs in bacteria (Fig. 3c). This extension of the biogenesis route is consistent with an evolutionarily conserved assembly mechanism.

Mitochondrial $\beta$-barrel OMPs must carry a signal that is recognized by the assembly machinery in the outer membrane. This signal, termed the $\beta$-signal, was recently identified by Kutik et al. (2008). Like the C-terminal signature sequence in bacterial OMPs described above, this $\beta$-signal is located near the $\mathrm{C}$ terminus of the OMPs. However, it is never located at the very end and is always followed by another 1-28 residues. As shown in Table 1 , the bacterial and mitochondrial signals, although not identical, appear to be rather similar and are probably evolutionarily related. Curiously, whereas the bacterial OMP signature sequence is recognized by the conserved central component BamA/Omp85 of the assembly machinery (Robert et al., 2006), the $\beta$-signal in the mitochondrial OMPs appears to be recognized by the accessory component Tob38 (Kutik et al., 2008). It should be noted, however, that the N-terminal POTRA domain of Tob55 has also been reported to interact with substrate proteins (Habib et al., 2007).

\section{Comparison of $\beta$-barrel OMP assembly in bacteria and mitochondria}

Comparison of $\beta$-barrel OMP assembly in bacteria and mitochondria reveals several similarities but also considerable differences. Firstly, the substrates in both cases are $\beta$-barrel proteins. However, while all bacterial OMPs

Table 1. Comparison of the $\beta$-signal of mitochondrial OMPs and the C-terminal signature sequence of bacterial OMPs, which are recognized by their respective OMP assembly machineries

The $\beta$-signal of the mitochondrial porin VDAC from Neurospora crassa and the signature sequence of the bacterial porin PhoE from E. coli are included in the comparison as examples. The one-letter code for amino acids is used. X, Any amino acid; $\phi$, hydrophobic residue; $\pi$, polar residue; n, 1-28 residues. The mitochondrial $\beta$-signal is given in bold type.

\begin{tabular}{|c|c|c|c|c|c|c|c|c|c|c|c|c|}
\hline \multirow[b]{2}{*}{ Mitochondrial $\beta$-signal } & \multicolumn{12}{|c|}{ Sequence } \\
\hline & $\mathrm{X}$ & $\mathrm{X}$ & $\pi$ & $\mathrm{X}$ & G & $\mathbf{X}$ & $\mathrm{X}$ & $\phi$ & $\mathbf{X}$ & $\phi$ & $(\mathrm{X}) \mathrm{n}$ & \\
\hline Bacterial signature & $\mathrm{X}$ & $\phi$ & $\mathrm{X}$ & $\phi$ & $\mathrm{X}$ & $\phi$ & $\mathrm{X}$ & $\mathrm{Y} / \phi$ & $\pi$ & $\mathrm{F}$ & - & - \\
\hline VDAC & $\mathrm{T}$ & $\mathrm{H}$ & K & V & G & $\mathrm{T}$ & $S$ & $\mathrm{~F}$ & $\mathrm{~T}$ & $\mathrm{~F}$ & $\mathrm{E}$ & S \\
\hline PhoE & I & $\mathrm{V}$ & A & V & G & M & $\mathrm{T}$ & $\mathrm{Y}$ & Q & $\mathrm{F}$ & - & - \\
\hline
\end{tabular}


appear to contain an even number of $\beta$-strands (Koebnik et al., 2000), the only mitochondrial $\beta$-barrel OMP of which the structure has been solved, i.e. the voltagedependent anion channel VDAC or mitochondrial porin, is a 19-stranded $\beta$-barrel (Bayrhuber et al., 2008; Hiller et al., 2008; Ujwal et al., 2008). It is interesting to note that a mutant form of porin PhoE lacking the first $\mathrm{N}$ terminal $\beta$-strand has been reported to be functionally assembled, albeit inefficiently, into the E. coli outer membrane (Bosch et al., 1988), demonstrating that the bacterial Bam complex can deal with $\beta$-barrels with an odd number of strands. Secondly, the OMP assembly machineries contain a conserved central component, Omp85/BamA in bacteria and Tob55 in mitochondria. However, Tob55 is considerably smaller than its bacterial homologues. It contains only a single POTRA domain at its $\mathrm{N}$ terminus (Fig. 3c), while the bacterial proteins contain five of these domains (Fig. 3a and b). A deletion analysis in $N$. meningitidis, however, revealed that a mutant expressing an Omp85 variant with only a single POTRA domain was viable and assembled OMPs into the outer membrane with only slightly decreased efficiency in the case of larger OMPs (Bos et al. 2007b). Thirdly, the bacterial and mitochondrial machineries contain several accessory components, which, however, show no mutual homology. Fourthly, signals for recognition by the assembly machineries have been identified near the $\mathrm{C}$ termini of both bacterial and mitochondrial $\beta$-barrel OMPs. These signals are similar but not completely identical. Moreover, they are recognized by different components of the assembly machineries, i.e. by Omp85/ BamA in the bacterial system and by Tob38 in the mitochondrial system.

\section{A mitochondrial $\beta$-barrel OMP can be assembled into the bacterial outer membrane}

The similarities between the bacterial and mitochondrial $\beta$-barrel OMPs and their assembly machineries suggest a common evolutionary origin. However, as described above, there are also considerable differences between the systems. Therefore, it was of interest to determine whether a mitochondrial OMP can be assembled into the bacterial outer membrane. To test this possibility, VDAC of Neurospora crassa was genetically fused to a signal sequence to mediate transport across the bacterial inner membrane via the Sec system, and the construct was expressed in E. coli (Walther et al., 2010). Cell fractionations, protease-sensitivity assays and immunofluorescence microscopy showed that VDAC was assembled into the bacterial outer membrane where it formed functional pores. Furthermore, assembly into the outer membrane was dependent on the C-terminal $\beta$-signal in VDAC and on the expression of a functional E. coli BamA protein (Walther et al., 2010). These results demonstrated that the bacterial OMP assembly machinery can still deal with the $\beta$-barrel OMPs that evolved in mitochondria.

\section{Bacterial OMPs can be assembled into the mitochondrial outer membrane}

It was also of interest to determine whether the $\beta$-barrel OMP assembly machinery that evolved in mitochondria is still able to handle bacterial OMPs. This question was more complicated to address, since $\beta$-barrel OMPs in mitochondria first have to be taken up via the TOM complex before reaching the TOB complex from the right side of the membrane (Fig. 3c). The mitochondrial $\beta$-barrel OMPs do not contain a cleavable signal for their targeting to mitochondria but rather an uncleavable internal signal. The nature of this signal has not been characterized and may be dispersed over the entire polypeptide rather than being confined to a discrete segment (Walther \& Rapaport, 2009). Such a signal would be difficult to fuse genetically to a bacterial OMP. However, it was also proposed that $\beta$ barrel-specific structural elements are recognized by the mitochondrial import machinery (Walther \& Rapaport, 2009), in which case, bacterial OMPs might also be recognized. To test this possibility, porin PhoE of E. coli was expressed in Saccharomyces cerevisiae without its signal sequence, which would presumably lead the protein to the endoplasmic reticulum (Walther et al., 2009a). The protein was found to accumulate in the mitochondria of the yeast in a TOM-dependent manner. Similar results were obtained for a diverse set of other bacterial OMPs. Thus, apparently, the bacterial OMPs contain the appropriate signals to be taken up into mitochondria via the TOM complex. These results indicate that no eukaryote-specific import signals were required to evolve in mitochondrial $\beta$ barrel OMPs to ensure their import into mitochondria when, during endosymbiont evolution, their structural genes were transferred to the nucleus.

The accumulation of PhoE in the mitochondria was also dependent on a functional TOB complex. The protein was inserted into the mitochondrial outer membrane in its native trimeric state and it was detectable at the surface of intact mitochondria with PhoE-specific monoclonal antibodies that recognize conformational epitopes (Walther et al., 2009a). The efficiency of the assembly into the mitochondrial outer membrane was dependent on the expression level; at low expression levels, all PhoE detected was correctly assembled into the trimeric configuration, whereas at high expression levels considerable amounts of the protein also accumulated as aggregates, presumably in the mitochondrial intermembrane space (Walther et al., 2009a). Thus, apparently, the capacity of the TOB complex to deal with the heterologous substrate protein is limited. Assembly of PhoE into the mitochondrial outer membrane was also dependent on its C-terminal signature sequence; when the mutant PhoE protein lacking the $\mathrm{C}$-terminal Phe was expressed in S. cerevisiae, it was taken up into the mitochondria but it was not assembled into the outer membrane in its native trimeric state (Walther et al., 2009a). Thus, collectively, bacterial OMPs can be assembled into the mitochondrial outer membrane and this assembly depends on their C-terminal signature 
sequence and on the mitochondrial TOM and TOB complexes.

\section{Conclusions}

In recent years, much progress has been made in studies on the biogenesis of bacterial OMPs. This progress is mostly related to the identification of the components of the machinery that assemble these proteins into the outer membrane and also on the resolution of the structures of the periplasmic chaperones involved, some in complex with their substrate OMPs. Progress was also stimulated by the discovery of a similar machinery for the insertion of $\beta$ barrel OMPs into the mitochondrial outer membrane. The basic mechanism of OMP assembly is conserved to such an extent that a mitochondrial OMP can be assembled in vivo into the bacterial outer membrane, and vice versa, bacterial OMPs can be assembled into the mitochondrial outer membrane. It is likely that a similar mechanism operates in chloroplasts (Hsu \& Inoue, 2009). Thus, results in these fields will be mutually profitable. Mechanistic insight into the assembly process and the function of the individual components of either of these systems is still very limited. Much progress is to be expected in the near future from the resolution of the structures of the components or, perhaps, of the entire machineries and from the development of reconstituted systems with purified components to study the assembly process in vitro.

\section{Acknowledgements}

Work in my laboratory was supported by grants from the Netherlands Councils for Chemical Sciences (CW) and for Earth and Life Sciences (ALW) from the Netherlands Organization for Scientific Research (NWO), the European Community, and la Fondation de la Recherche Médicale. I gratefully acknowledge the contributions of present and former members of my research group to the work described, especially Martine Bos, Romé Voulhoux, Viviane Robert, Elena Volokhina, Hans de Cock and Marlies Struyvé. Special thanks are also due to Patrick Van Gelder for many valuable contributions through many years and to Doron Rapaport and Dirk Walther (University of Tübingen) for the collaborative work on the conservation of the mechanism of OMP assembly in bacteria and mitochondria. Finally, I would like to thank Frouke Kuijer for the preparation of the figures.

\section{References}

Agterberg, M., Adriaanse, H., van Bruggen, A., Karperien, M. \& Tommassen, J. (1990). Outer-membrane PhoE protein of Escherichia coli K-12 as an exposure vector: possibilities and limitations. Gene $\mathbf{8 8}$, 37-45.

Anwari, K., Poggio, S., Perry, A., Gatsos, X., Ramarathinam, S. H., Williamson, N. A., Noinaj, N., Buchanan, S., Gabriel, K. \& other authors (2010). A modular BAM complex in the outer membrane of the $\alpha$-proteobacterium Caulobacter crescentus. PLoS One 5, e8619.

Bayrhuber, M., Meins, T., Habeck, M., Becker, S., Giller, K., Villinger, S., Vonrhein, C., Griesinger, C., Zweckstetter, M. \& Zeth, K. (2008). Structure of the human voltage-dependent anion channel. Proc Natl Acad Sci U S A 105, 15370-15375.
Behrens, S., Maier, R., de Cock, H., Schmid, F. X. \& Gross, C. A. (2001). The SurA periplasmic PPIase lacking its parvulin domains functions in vivo and has chaperone activity. EMBO J 20, 285-294.

Bos, M. P., Tefsen, B., Geurtsen, J. \& Tommassen, J. (2004). Identification of an outer membrane protein required for lipopolysaccharide transport to the bacterial cell surface. Proc Natl Acad Sci U S A 101, 9417-9422.

Bos, M. P., Robert, V. \& Tommassen, J. (2007a). Biogenesis of the Gram-negative bacterial outer membrane. Annu Rev Microbiol 61, 191-214.

Bos, M. P., Robert, V. \& Tommassen, J. (2007b). Functioning of outer membrane protein assembly factor Omp85 requires a single POTRA domain. EMBO Rep 8, 1149-1154.

Bosch, D., Voorhout, W. \& Tommassen, J. (1988). Export and localization of N-terminally truncated derivatives of Escherichia coli K-12 outer membrane protein PhoE. J Biol Chem 263, 9952-9957.

Chacinska, A., Koehler, C. A., Milenkovic, D., Lithgow, T. \& Pfanner, N. (2009). Importing mitochondrial proteins: machineries and mechanisms. Cell 138, 628-644.

Chen, R. \& Henning, U. (1996). A periplasmic protein (Skp) of Escherichia coli selectively binds a class of outer membrane proteins. Mol Microbiol 19, 1287-1294.

Costanzo, A. \& Ades, S. E. (2006). Growth phase-dependent regulation of the extracytoplasmic stress factor, $\sigma^{\mathrm{E}}$, by guanosine 3',5'-bipyrophosphate (ppGpp). J Bacteriol 188, 4627-4634.

de Cock, H., Struyvé, M., Kleerebezem, M., van der Krift, T. \& Tommassen, J. (1997). Role of the carboxy-terminal phenylalanine in the biogenesis of outer membrane protein PhoE of Escherichia coli K12. J Mol Biol 269, 473-478.

de Cock, H., Schäfer, U., Potgeter, M., Demel, R., Müller, M. \& Tommassen, J. (1999). Affinity of the periplasmic chaperone Skp of Escherichia coli for phospholipids, lipopolysaccharides and non-native outer membrane proteins. Role of Skp in the biogenesis of outer membrane protein. Eur J Biochem 259, 96-103.

Dekker, N., Merck, K., Tommassen, J. \& Verheij, H. M. (1995). In vitro folding of Escherichia coli outer-membrane phospholipase A. Eur J Biochem 232, 214-219.

Driessen, A. J. M. \& Nouwen, N. (2008). Protein translocation across the bacterial cytoplasmic membrane. Annu Rev Biochem 77, 643-667.

Fardini, Y., Trotereau, J., Bottreau, E., Souchard, C., Velge, P. \& Virlogeux-Payant, I. (2009). Investigation of the role of the BAM complex and SurA chaperone in outer membrane protein biogenesis and T3SS expression in Salmonella. Microbiology 155, 1613-1622.

Fussenegger, M., Facius, D., Meier, J. \& Meyer, T. F. (1996). A novel peptidoglycan-linked lipoprotein (ComL) that functions in natural transformation competence of Neisseria gonorrhoeae. Mol Microbiol 19, 1095-1105.

Gatsos, X., Perry, A. J., Anwari, K., Dolezal, P., Wolynec, P. P., Likić, V. A., Purcell, A. W., Buchanan, S. K. \& Lithgow, T. (2008). Protein secretion and outer membrane assembly in Alphaproteobacteria. FEMS Microbiol Rev 32, 995-1009.

Gatzeva-Topalova, P. Z., Walton, T. A. \& Sousa, M. C. (2008). Crystal structure of YaeT: conformational flexibility and substrate recognition. Structure 16, 1873-1881.

Gentle, I., Gabriel, K., Beech, P., Waller, R. \& Lithgow, T. (2004). The Omp85 family of proteins is essential for outer membrane biogenesis in mitochondria and bacteria. J Cell Biol 164, 19-24.

Habib, S. J., Waizenegger, T., Niewienda, A., Paschen, S. A., Neupert, W. \& Rapaport, D. (2007). The N-terminal domain of Tob55 has a receptor-like function in the biogenesis of mitochondrial $\beta$-barrel proteins. J Cell Biol 176, 77-88. 
Harms, N., Koningstein, G., Dontje, W., Müller, M., Oudega, B., Luirink, J. \& de Cock, H. (2001). The early interaction of the outer membrane protein PhoE with the periplasmic chaperone Skp occurs at the cytoplasmic membrane. J Biol Chem 276, 18804-18811.

Hiller, S., Garces, R. G., Malia, T. J., Orekhov, V. Y., Colombini, M. \& Wagner, G. (2008). Solution structure of the integral human membrane protein VDAC-1 in detergent micelles. Science 321, $1206-1210$.

Hsu, S.-C. \& Inoue, K. (2009). Two evolutionarily conserved essential $\beta$-barrel proteins in the chloroplast outer envelope membrane. Biosci Trends 3, 168-178.

Ishikawa, D., Yamamoto, H., Tamura, Y., Moritoh, K. \& Endo, T. (2004). Two novel proteins in the mitochondrial outer membrane mediate $\beta$-barrel protein assembly. J Cell Biol 166, 621-627.

Johansen, J., Rasmussen, A. A., Overgaard, M. \& Valentin-Hansen, P. (2006). Conserved small non-coding RNAs that belong to the $\sigma^{\mathrm{E}}$ regulon: role in down-regulation of outer membrane proteins. $J \mathrm{Mol}$ Biol 364, 1-8.

Kim, S., Malinverni, J. C., Sliz, P., Silhavy, T. J., Harrison, S. C. \& Kahne, D. (2007). Structure and function of an essential component of the outer membrane protein assembly machine. Science $317,961-$ 964.

Knowles, T. J., Jeeves, M., Bobat, S., Dancea, F., McClelland, D., Palmer, T., Overduin, M. \& Henderson, I. R. (2008). Fold and function of polypeptide transport-associated domains responsible for delivering unfolded proteins to membranes. Mol Microbiol 68, 1216-1227.

Koebnik, R., Locher, K. P. \& Van Gelder, P. (2000). Structure and function of bacterial outer membrane proteins: barrels in a nutshell. Mol Microbiol 37, 239-253.

Korndörfer, I. P., Dommel, M. K. \& Skerra, A. (2004). Structure of the periplasmic chaperone Skp suggests functional similarity with cytosolic chaperones despite differing architecture. Nat Struct Mol Biol 11, 1015-1020.

Kozjak, V., Wiedemann, N., Milenkovic, D., Lohaus, C., Meyer, H. E., Guiard, B., Meisinger, C. \& Pfanner, N. (2003). An essential role of Sam50 in the protein sorting and assembly machinery of the mitochondrial outer membrane. J Biol Chem 278, 48520-48523.

Krimmer, T., Rapaport, D., Ryan, M. T., Meisinger, C., Kassenbrock, C. K., Blachly-Dyson, E., Forte, M., Douglas, M. G., Neupert, W. \& other authors (2001). Biogenesis of the major mitochondrial outer membrane protein porin involves a complex import pathway via receptors and the general import pore. J Cell Biol 152, 289-300.

Krojer, T., Sawa, J., Schäfer, E., Saibil, H. R., Ehrmann, M. \& Clausen, T. (2008). Structural basis for the regulated protease and chaperone function of DegP. Nature 453, 885-890.

Kutik, S., Stojanovski, D., Becker, L., Becker, T., Meinecke, M., Krüger, V., Prinz, C., Meisinger, C., Guiard, B. \& other authors (2008). Dissecting membrane insertion of mitochondrial $\beta$-barrel proteins. Cell 132, 1011-1024.

Lazar, S. W. \& Kolter, R. (1996). SurA assists the folding of Escherichia coli outer membrane proteins. J Bacteriol 178, 1770-1773.

Malinverni, J. C., Werner, J., Kim, S., Sklar, J. G., Kahne, D., Misra, R. \& Silhavy, T. J. (2006). YfiO stabilizes the YaeT complex and is essential for outer membrane protein assembly in Escherichia coli. Mol Microbiol 61, 151-164.

Manning, D. S., Reschke, D. K. \& Judd, R. C. (1998). Omp85 of Neisseria gonorrhoeae and Neisseria meningitidis are similar to Haemophilus influenzae D-15-Ag and Pasteurella multocida Oma87. Microb Pathog 25, 11-21.

Milenkovic, D., Kozjak, V., Wiedemann, N., Lohaus, C., Meyer, H. E., Guiard, B., Pfanner, N. \& Meisinger, C. (2004). Sam35 of the mitochondrial protein sorting and assembly machinery is a peripheral outer membrane protein essential for cell viability. J Biol Chem 279, 22781-22785.

Model, K., Meisinger, C., Prinz, T., Wiedemann, N., Truscott, K. N., Pfanner, N. \& Ryan, M. T. (2001). Multistep assembly of the protein import channel of the mitochondrial outer membrane. Nat Struct Biol 8, 361-370.

Nakamura, K. \& Mizushima, S. (1976). Effects of heating in dodecyl sulfate solution on the conformation and electrophoretic mobility of isolated major outer membrane proteins from Escherichia coli K-12. J Biochem 80, 1411-1422.

Nikaido, H. (2003). Molecular basis of bacterial outer membrane permeability revisited. Microbiol Mol Biol Rev 67, 593-656.

Papenfort, K., Pfeiffer, V., Mika, F., Lucchini, S., Hinton, J. C. D. \& Vogel, J. (2006). $\sigma^{\mathrm{E}}$-dependent small RNAs of Salmonella respond to membrane stress by accelerating global omp mRNA decay. Mol Microbiol 62, 1674-1688.

Paschen, S. A., Waizenegger, T., Stan, T., Preuss, M., Cyrklaff, M., Hell, K., Rapaport, D. \& Neupert, W. (2003). Evolutionary conservation of biogenesis of $\beta$-barrel membrane proteins. Nature 426, 862866.

Rapaport, D. \& Neupert, W. (1999). Biogenesis of Tom40, core component of the TOM complex of mitochondria. J Cell Biol 146, 321-331.

Reumann, S., Davila-Aponte, J. \& Keegstra, K. (1999). The evolutionary origin of the protein-translocating channel of chloroplastic envelope membranes: Identification of a cyanobacterial homolog. Proc Natl Acad Sci U S A 96, 784-789.

Rizzitello, A. E., Harper, J. R. \& Silhavy, T. J. (2001). Genetic evidence for parallel pathways of chaperone activity in the periplasm of Escherichia coli. J Bacteriol 183, 6794-6800.

Robert, V., Volokhina, E. B., Senf, F., Bos, M. P., Van Gelder, P. \& Tommassen, J. (2006). Assembly factor Omp85 recognizes its outer membrane protein substrates by a species-specific C-terminal motif. PLoS Biol 4, e377.

Rouvière, P. E. \& Gross, C. A. (1996). SurA, a periplasmic protein with peptidyl-prolyl isomerase activity, participates in the assembly of outer membrane porins. Genes Dev 10, 3170-3187.

Ruiz, N. \& Silhavy, T. J. (2005). Sensing external stress: watchdogs of the Escherichia coli cell envelope. Curr Opin Microbiol 8, 122-126.

Rutten, L., Mannie, J.-P. B. A., Stead, C. M., Raetz, C. R. H., Reynolds, C. M., Bonvin, A. M. J. J., Tommassen, J. P., Egmond, M. R., Trent, M. S. \& Gros, P. (2009). Active-site architecture and catalytic mechanism of the lipid A deacylase LpxR of Salmonella typhimurium. Proc Natl Acad Sci U S A 106, 1960-1964.

Sánchez-Pulido, L., Devos, D., Genevrois, S., Vicente, M. \& Valencia, A. (2003). POTRA: a conserved domain in the FtsQ family and a class of $\beta$ barrel outer membrane proteins. Trends Biochem Sci 28, 523-526.

Schäfer, U., Beck, K. \& Müller, M. (1999). Skp, a molecular chaperone of Gram-negative bacteria, is required for the formation of soluble periplasmic intermediates of outer membrane proteins. J Biol Chem 274, 24567-24574.

Sklar, J. G., Wu, T., Gronenberg, L. S., Malinverni, J. C., Kahne, D. \& Silhavy, T. J. (2007a). Lipoprotein SmpA is a component of the YaeT complex that assembles outer membrane proteins in Escherichia coli. Proc Natl Acad Sci U S A 104, 6400-6405.

Sklar, J. G., Wu, T., Kahne, D. \& Silhavy, T. J. (2007b). Defining the roles of the periplasmic chaperones SurA, Skp, and DegP in Escherichia coli. Genes Dev 21, 2473-2484.

Spiess, C., Beil, A. \& Ehrmann, M. (1999). A temperature-dependent switch from chaperone to protease in a widely conserved heat shock protein. Cell 97, 339-347. 
Steeghs, L., den Hartog, R., den Boer, A., Zomer, B., Roholl, P. \& van der Ley, P. (1998). Meningitis bacterium is viable without endotoxin. Nature 392, 449-450.

Steeghs, L., de Cock, H., Evers, E., Zomer, B., Tommassen, J. \& van der Ley, P. (2001). Outer membrane composition of a lipopolysaccharide-deficient Neisseria meningitidis mutant. EMBO J 20, 69376945 .

Stegmeier, J. F. \& Andersen, C. (2006). Characterization of pores formed by YaeT (Omp85) from Escherichia coli. J Biochem 140, 275-283.

Struyvé, M., Moons, M. \& Tommassen, J. (1991). Carboxy-terminal phenylalanine is essential for the correct assembly of a bacterial outer membrane protein. J Mol Biol 218, 141-148.

Tam, C. \& Missiakas, D. (2005). Changes in lipopolysaccharide structure induce the $\sigma^{\mathrm{E}}$-dependent response of Escherichia coli. Mol Microbiol 55, 1403-1412.

Tefsen, B., Bos, M. P., Beckers, F., Tommassen, J. \& de Cock, H. (2005). MsbA is not required for phospholipid transport in Neisseria meningitidis. J Biol Chem 280, 35961-35966.

Thomas, K. L., Leduc, I., Olsen, B., Thomas, C. E., Cameron, D. W. \& Elkins, C. (2001). Cloning, overexpression, purification, and immunobiology of an 85-kilodalton outer membrane protein from Haemophilus ducreyi. Infect Immun 69, 4438-4446.

Tsukazaki, T., Mori, H., Fukai, S., Ishitani, R., Mori, T., Dohmae, N., Perederina, A., Sugita, Y., Vassylyev, D. G. \& other authors (2008). Conformational transition of Sec machinery inferred from bacterial SecYE structures. Nature 455, 988-992.

Ujwal, R., Cascio, D., Colletier, J. P., Faham, S., Zhang, J., Toro, L., Ping, P. \& Abramson, J. (2008). The crystal structure of mouse VDAC1 at $2.3 \AA$ resolution reveals mechanistic insights into metabolite gating. Proc Natl Acad Sci U S A 105, 17742-17747.

Vertommen, D., Ruiz, N., Leverrier, P., Silhavy, T. J. \& Collet, J.-F. (2009). Characterization of the role of the Escherichia coli periplasmic chaperone SurA using differential proteomics. Proteomics 9, 2432-2443.

Volokhina, E. B., Beckers, F., Tommassen, J. \& Bos, M. P. (2009). The $\beta$-barrel outer membrane protein assembly complex of Neisseria meningitidis. J Bacteriol 191, 7074-7085.
Voulhoux, R. \& Tommassen, J. (2004). Omp85, an evolutionarily conserved bacterial protein involved in outer-membrane-protein assembly. Res Microbiol 155, 129-135.

Voulhoux, R., Bos, M. P., Geurtsen, J., Mols, M. \& Tommassen, J. (2003). Role of a highly conserved bacterial protein in outer membrane protein assembly. Science 299, 262-265.

Waizenegger, T., Habib, S. J., Lech, M., Mokranjac, D., Paschen, S. A., Hell, K., Neupert, W. \& Rapaport, D. (2004). Tob38, a novel essential component in the biogenesis of $\beta$-barrel proteins of mitochondria. EMBO Rep 5, 704-709.

Walther, D. M. \& Rapaport, D. (2009). Biogenesis of mitochondrial outer membrane proteins. Biochim Biophys Acta 1793, 42-51.

Walther, D. M., Papic, D., Bos, M. P., Tommassen, J. \& Rapaport, D. (2009a). Signals in bacterial $\beta$-barrel proteins are functional in eukaryotic cells for targeting to and assembly in mitochondria. Proc Natl Acad Sci U S A 106, 2531-2536.

Walther, D. M., Rapaport, D. \& Tommassen, J. (2009b). Biogenesis of $\beta$-barrel membrane proteins in bacteria and eukaryotes: evolutionary conservation and divergence. Cell Mol Life Sci 66, 2789-2804.

Walther, D. M., Bos, M. P., Rapaport, D. \& Tommassen, J. (2010). The mitochondrial porin, VDAC, has retained the ability to be assembled in the bacterial outer membrane. Mol Biol Evol 27, 887-895.

Walton, T. A. \& Sousa, M. C. (2004). Crystal structure of Skp, a prefoldin-like chaperone that protects soluble and membrane proteins from aggregation. Mol Cell 15, 367-374.

Walton, T. A., Sandoval, C. M., Fowler, C. A., Pardi, A. \& Sousa, M. C. (2009). The cavity-chaperone Skp protects its substrate from aggregation but allows independent folding of substrate domains. Proc Natl Acad Sci U S A 106, 1772-1777.

Wiedemann, N., Kozjak, V., Chacinska, A., Schönfish, B., Rospert, S., Ryan, M. T., Pfanner, N. \& Meisinger, C. (2003). Machinery for protein sorting and assembly in the mitochondrial outer membrane. Nature 424, 565-571.

Wu, T., Malinverni, J., Ruiz, N., Kim, S., Silhavy, T. J. \& Kahne, D. (2005). Identification of a multicomponent complex required for outer membrane biogenesis in Escherichia coli. Cell 121, 235-245. 